\title{
Correction to: Strength and dilatancy of coral sand in the South China Sea
}

\author{
Xing Wang ${ }^{1} \cdot$ Yongxin $\mathrm{Wu}^{2} \cdot \mathrm{Yi} \mathrm{Lu}^{1} \cdot$ Jie Cui ${ }^{1} \cdot \mathrm{Xinzhi} \mathrm{Wang}^{3} \cdot$ Changqi Zhu $^{3}$
}

Published online: 10 August 2021

๑) Springer-Verlag GmbH Germany, part of Springer Nature 2021

Correction to: Bulletin of Engineering Geology and the Environment (2021) https://doi.org/10.1007/s10064-021-02348-6

In the online published article, there are the following errors.

1. Equations 1,2 and 5 are incorrect.

2. In Equation 22, there are two " $="$ signs.

3. The symbol' 'needs to be replaced by the symbol ' wherever (i.e. in equations and in the text) it appears.

4. The symbols on the left hand side of the Equations 4 and 9 should be in Italics.

5. There are errors in the first row of Tables 3 and 4 .
The original article has been corrected.

The original article can be found online at https://doi.org/10.1007/ s10064-021-02348-6.

Yi Lu

luyi@gzhu.edu.cn

1 School of Civil Engineering, Guangzhou University, Guangzhou 510006, China

2 Key Laboratory of Ministry of Education for Geomechanics and Embankment Engineering, Hohai University, Nanjing 210024, China

3 State Key Laboratory of Geotechnical Mechanics and Engineering, Institute of Rock and Soil Mechanics, Chinese Academy of Sciences, Wuhan 430071, China 